\title{
THE INERTIAL CAVITATION THRESHOLD IN SOFT TISSUE USING A DUAL-FREQUENCY DRIVING SIGNAL
}

\author{
TATIANA FILONETS ${ }^{1,2}$, MAXIM SOLOVCHUK ${ }^{1,2}$ AND TONY W.H. SHEU ${ }^{3}$ \\ ${ }^{1}$ Department of Engineering Science and Ocean Engineering, National Taiwan University \\ No. 1, Sec.4, Roosevelt Road, Taipei 10617, Taiwan, ROC \\ tfilonets@gmail.com \\ ${ }^{2}$ Institute of Biomedical Engineering and Nanomedicine, National Health Research Institutes \\ No. 35, Keyan Road, Zhunan 35053, Taiwan, ROC \\ solovchuk@gmail.com \\ ${ }^{3}$ Department of Engineering Science and Ocean Engineering, National Taiwan University \\ No. 1, Sec.4, Roosevelt Road, Taipei 10617, Taiwan, ROC \\ twhsheu@ntu.edu.tw
}

Key words: High intensity focused ultrasound, Acoustic cavitation in soft tissue, Inertial cavitation threshold, Dual-frequency signal, GPU programming

\begin{abstract}
High Intensity Focused Ultrasound (HIFU) is a non-invasive technology that can be applied for treatment of different diseases and ablation of tumours in different parts of the body. When high intensity ultrasound propagates through the medium bubbles can be formed, a phenomenon known as acoustic cavitation. There are two different regimes of acoustic cavitation: stable cavitation when a bubble just oscillates around an equilibrium state, and inertial cavitation which is accompanied by bubble collapse. These two different regimes can be used for different biomedical applications. However, in some cases it can also make the treatment less predictable. Therefore, fundamental understanding of these effects is very important. In the current study theoretical investigation of the bubble dynamics in viscoelastic medium is performed and inertial cavitation thresholds have been calculated. To describe the bubble dynamics, Gilmore-Akulichev-Zener model has been used, which is suitable for a large bubble oscillations and high ultrasound powers. The results showed that using the dual-frequency driving signal the threshold value of inertial cavitation can be significantly reduced compared to single-frequency signal mode. Large difference between frequencies in the dual-frequency signal leads to lower threshold values. Numerical simulations also showed the dependencies of the cavitation threshold on the bubble radius.
\end{abstract}




\section{INTRODUCTION}

High-intensity focused ultrasound (HIFU) therapy has been applied to treat cancer in different organs [1]. It can can be also applied for acoustic hemostasis which helps to seal a bleeding site [2]. At high negative pressures and high temperatures bubbles can be formed in the medium [3]. This process is called acoustic cavitationa and it often appears during HIFU treatment [4].

The cavitation process can enhance the energy delivery during HIFU therapy, it can also help in drug and gene delivery. At low ultrasound pressures, bubbles experience stable oscillations. When pressure reaches some critical threshold value, bubbles start to collapse. Bubble collapse is accompanied by a big increase in temperature inside the medium and correspondingly in larger ablated volume [4,5]. This type of cavitation is called inertial cavitation, and, therefore, its threshold is called inertial cavitation threshold. Another type of cavitation is called stable cavitation when bubbles just pulse about their equilibrium radius [5]. Stable cavitation bubbles can generate some effects such as surface wave activity and microstreaming [6]. Also, stable cavitation gives an opportunity to study, for example, multibubble sonoluminescence [7].

In this paper we will focus on inertial cavitation since it is more dangerous and can brings additional damages to the tissue. The inertial cavitation threshold depends on many parameters such as medium properties (density, viscosity, elasticity, and etc.), initial bubble size, and type of ultrasound signal $[8,9]$. Few recent theoretical studies [9-11] demonstrated that using dual-frequency driven signal is capable to reduce the inertial cavitation threshold in comparison with single-frequency signal mode and with using the same power output [9].

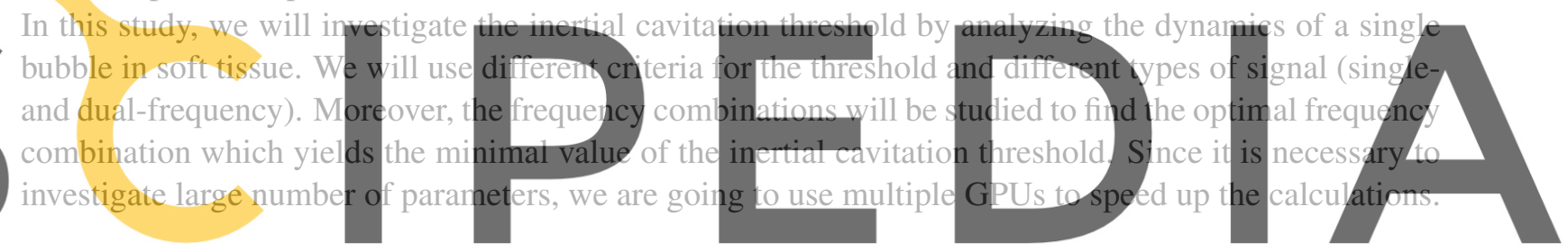

2 CAVITATION MODELS

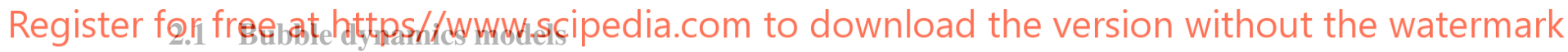

In order to numerically investigate bubble dynamics and inertial cavitation threshold in a viscoelastic medium during HIFU therapy, in the present study Gilmore-Akulichev-Zener model $[12,13]$ will be used.

Zener model is a more general linear viscoelastic model since it takes into consideration all three tissue parameters: viscosity $\mu$, elasticity $G$, and relaxation time $\lambda$ [14]:

$$
\lambda \dot{\tau}_{r r}+\tau=2 G \gamma_{r r}+2 \mu \gamma_{r r}
$$

where $\tau_{r r}$ is the stress and $\gamma_{r r}$ is the strain in $r$ direction ( $r$ is the distance from the bubble center). Moreover, from equation (1) we also can obtain other well-known viscoelastic models [14] such as Kelvin-Voight model (when $\lambda=0$ ) or Maxwell model (when $G=0$ ).

Keller-Miksis model is one of the most frequently used model for the description of bubble dynamics [14-17]:

$$
\left(1-\frac{\dot{R}}{c_{\infty}}\right) R \ddot{R}+\frac{3}{2}\left(1-\frac{1}{3} \frac{\dot{R}}{c_{\infty}}\right) \dot{R}^{2}=\left(1+\frac{\dot{R}}{c_{\infty}}\right) \frac{p_{B}-p_{\infty}-p_{A}(t)}{\rho_{\infty}}+\frac{R}{\rho_{\infty} c_{\infty}} \dot{p}_{B}
$$




$$
p_{B}=p_{i}-\frac{2 \sigma}{R}+3 q, \quad q=\int_{R}^{\infty} \frac{\tau_{r r}(t, r)}{r} d r, \quad p_{i}=p_{G}\left(\frac{R_{0}}{R}\right)^{3 \gamma}, \quad p_{G}=p_{\infty}+\frac{2 \sigma}{R_{0}},
$$

where $R$ is a bubble radius, the time derivative is denoted by the dot, $c_{\infty}$ is sound speed, $\rho_{\infty}$ is the environmental density, $p_{\infty}$ is the far-field pressure, $p_{A}$ is the time-varying sound field, $p_{B}$ is the pressure from the surrounding medium on the bubble interface, $p_{i}$ is the internal pressure of the bubble, $\sigma$ is the surface tension, $R_{0}$ is the initial bubble radius, $\gamma$ is the polytropic index (we assume adiabatic behavior here), $p_{G}$ is the initial pressure of non-condensible gas inside the bubble.

Keller-Miksis model can be suitable for the cases with large-amplitude oscillations, for example, for the inertial cavitation. Integrating equation (1) from $R$ to infinity

$$
\lambda \dot{q}+q+\left.\lambda \frac{\dot{R}}{R} \tau_{r r}\right|_{R}=\frac{1}{3}\left[-\frac{4}{3} G\left(1-\frac{R_{0}^{3}}{R^{3}}\right)-4 \mu \frac{\dot{R}}{R}\right],
$$

and evaluating equation (1) at $r=R$

$$
\left.\lambda \dot{\tau}_{r r}\right|_{R}+\left.\tau_{r r}\right|_{R}=-\frac{4}{3} G\left(1-\frac{R_{0}^{3}}{R^{3}}\right)-4 \mu \frac{\dot{R}}{R}
$$

Keller-Miksis-Zener model (KMZ) is obtained [14] which is described by the closed system of equations (2)-(3) and (4)-(5).

However, KMZ model is only correct up to Mach numbers smaller than 1 [16]. Another model, suggested by Gilmore and Akulichev [18-20], can be applied for larger amplitudes of the bubble oscillations [21].

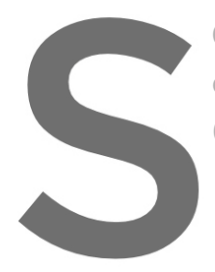
Combining Gilmore-Al of bubble dynamics in (GAZ) model and has the
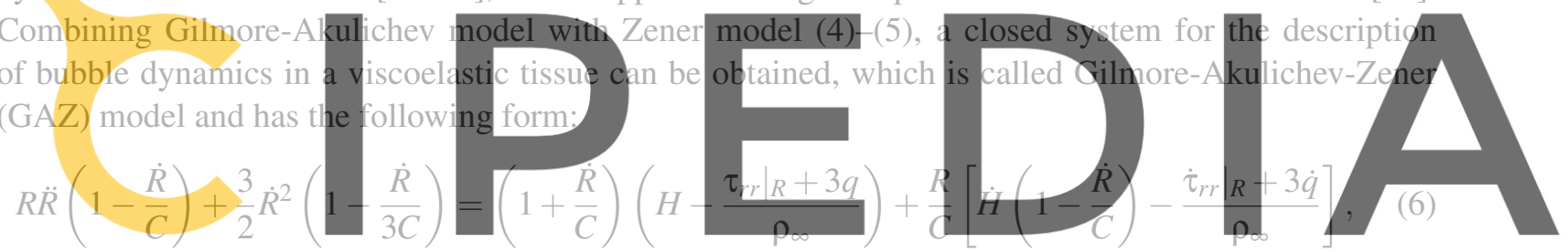

Register for free at https//www.scipedia.com to downdload the version without the watermark

$$
\begin{gathered}
H=\frac{1}{p_{\infty}} \frac{n}{n-1}\left(p_{\infty}+p_{A}(t)+B\right)\left(\frac{p_{i}-\frac{2 \sigma}{R}+\tau_{r r}+B}{p_{\infty}+p_{A}(t)+B}\right) \\
C=c_{\infty}\left(\frac{p_{i}-\frac{2 \sigma}{R}+\left.\tau_{r r}\right|_{R}+B}{p_{\infty}+p_{A}(t)+B}\right)^{\frac{n-1}{2 n}},
\end{gathered}
$$

where $H$ is an enthalpy at the bubble wall, $C$ is speed of sound at the bubble wall, $B$ and $n$ are special constants of the surrounding medium $[12,13]$. Please refer to [12] for a detailed derivation of GAZ model.

\subsection{Acoustic model}

To model the wave propagation in a viscoelastic medium, nonlinear Westervelt equation is used:

$$
\nabla^{2} p_{A}-\frac{1}{c_{\infty}^{2}} \frac{\partial^{2} p_{A}}{\partial t^{2}}+\frac{\delta}{c_{\infty}{ }^{4}} \frac{\partial^{3} p_{A}}{\partial t^{3}}+\frac{\beta}{\rho_{\infty} c_{\infty}{ }^{4}} \frac{\partial^{2} p_{A}}{\partial t^{2}}=0
$$


where $p_{A}$ is pressure of the sound field, $\delta$ is the sound diffusion, $\beta$ is the coefficient of nonlinearity. In the current paper, we will use only linear wave propagation. Therefore, equation (9) takes the following form:

$$
\nabla^{2} p_{A}-\frac{1}{c_{\infty}^{2}} \frac{\partial^{2} p_{A}}{\partial t^{2}}+\frac{\delta}{c_{\infty}^{4}} \frac{\partial^{3} p_{A}}{\partial t^{3}}=0
$$

The effects of nonlinear propagation can be included in the model by using nonlinear Westervelt equation (9), as it was shown in previous papers of the present authors [22,23].

In the current study for clarity sake, the single-frequency acoustic signal is used $p_{A}(t)=A \cos \left(2 \pi f_{1} t\right)$, whereas for the dual-frequency acoustic signal we use

$$
p_{A}(t)=\frac{A}{\sqrt{2}}\left[\cos \left(2 \pi f_{1} t\right)+\cos \left(2 \pi f_{2} t\right)\right]
$$

where $A$ is the acoustic pressure amplitude, $f_{1}$ and $f_{2}$ are the driving acoustic frequencies. Additional coefficient $1 / \sqrt{2}$ in equation (11) is necessary to ensure the same output power delivered as that of the single-frequency signal [9].

\subsection{Inertial cavitation threshold}

There are different definitions of the criteria for the inertial cavitation [9,24-26]. One of the popular and commonly used definitions of the inertial cavitation threshold is doubled bubble expansion in relation to the initial bubble size, $R$

used as the inertial cavi speed of sound.

In the current study, the in

of $1 \mathrm{kPa}$, for different modes: single- and dual-f
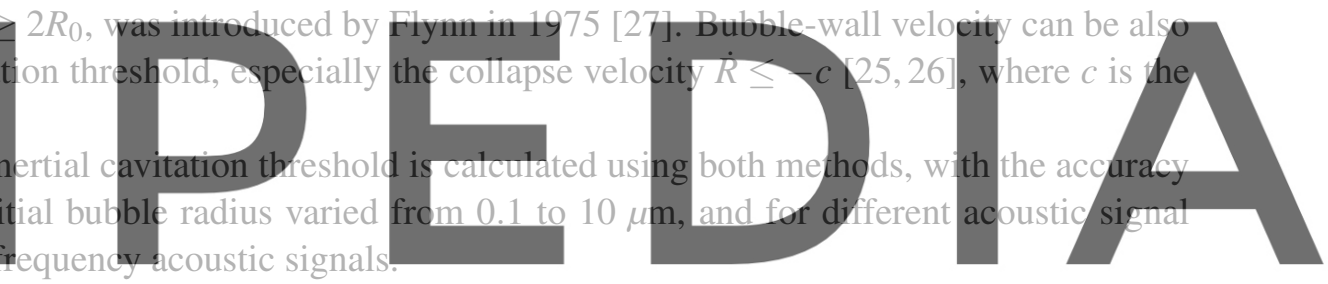

Since the bubble dynamics in soft tissue is investigated, liver properties have been used. Values of all

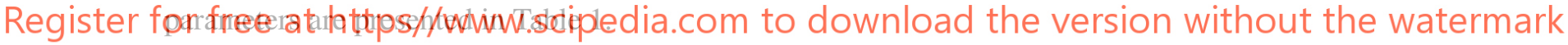

\section{NUMERICAL RESULTS}

To solve the systems of equations for GAZ and KMZ models, the Runge-Kutta-Dormand-Prince numerical method was used with step size control and with an absolute and relative error of $10^{-9}$ [28]. This method allows to construct the fifth-order solution. We wrote a program in $\mathrm{C}++$ to build the dynamics of the bubble. Due to a large number of investigated parameters in order to find the threshold of inertial cavitation, we used parallel calculations on graphical processor units (4 GPUs type of Titan V) for high performance and speed up of our calculations.

\subsection{Comparison of bubble dynamics models}

To test our calculations of bubble dynamics, we compared the obtained results (Figure 1) with results from Zilonova et al [12], where the difference between the GAZ and KMZ models also was demonstrated.

Figure 1 shows the solutions of the GAZ and KMZ equations for small (Figure 1a), medium (Figure 1b), and high (Figure 1c) amplitude values of the acoustic signal. As in [12], at low amplitudes, the GAZ and 
Table 1: Values of the model parameters

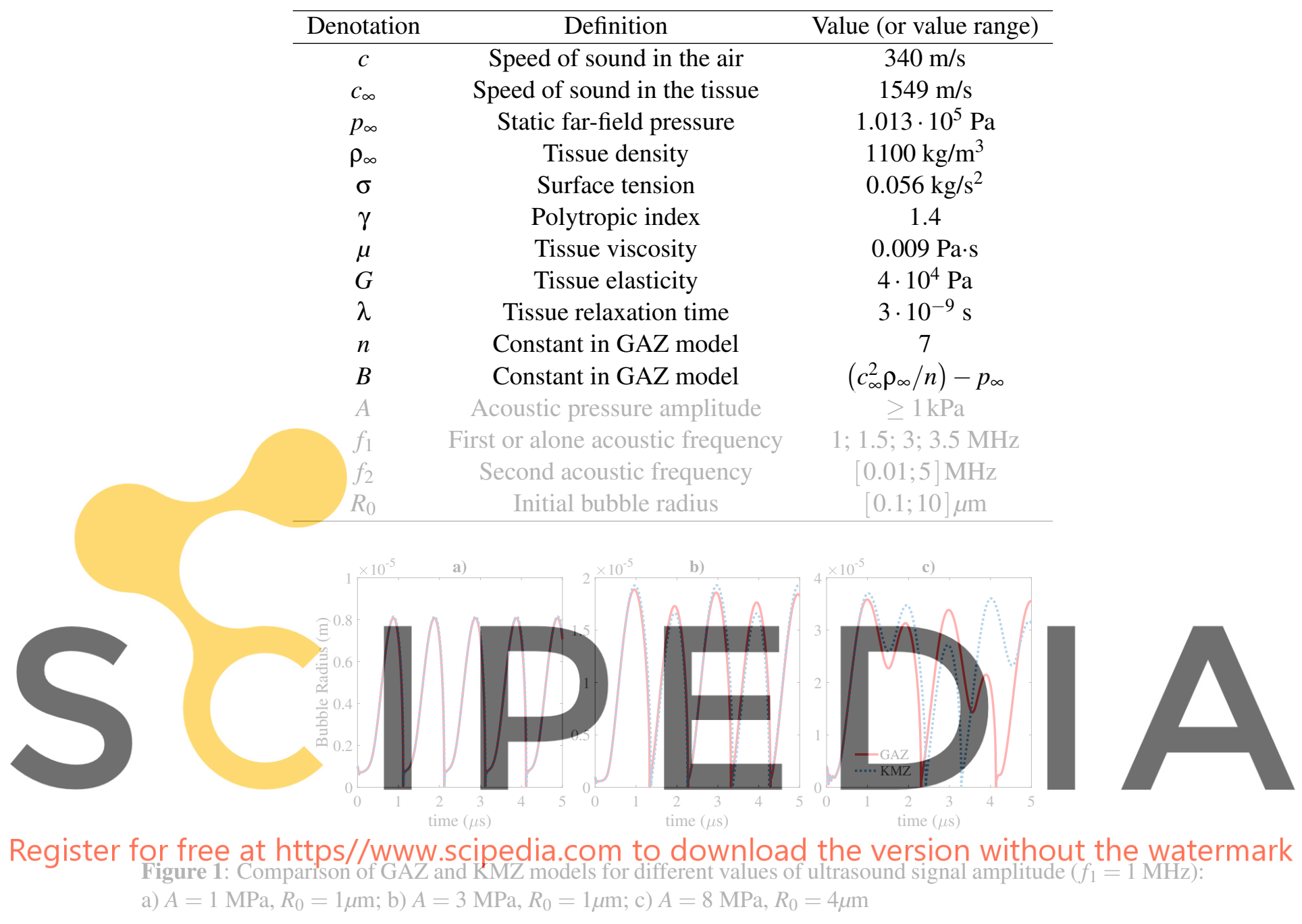

KMZ models give similar results (Figure 1a-b). At high amplitudes, the difference in results of GAZ and KMZ models becomes significant: different maximum values of the bubble radius, as well as different bubble collapse time (Figure 1c). These differences are explained by the fact that the Gilmore-Akulichev model can be applied for Mach numbers greater than one (and less than 2.2 [12]), while the Keller-Miksis model can only be used for Mach numbers less than one. Therefore, in this paper, we will use the GAZ model for further investigation of the inertial cavitation threshold.

\subsection{Comparison of single- and dual-frequency signal modes}

When a second wave is added to the dual-frequency mode of an acoustic signal, the behavior of this signal becomes not as stable as in the single-frequency mode: new peaks and new attenuations of signal appear (Figure 2a-b). At the same time, the increase in the bubble radius values during the dual-frequency signal mode is stronger than during the single-frequency mode, especially with the use of high frequencies 
(Figure 2d). When high frequencies are used, the influence of the dual-frequency signal mode on the
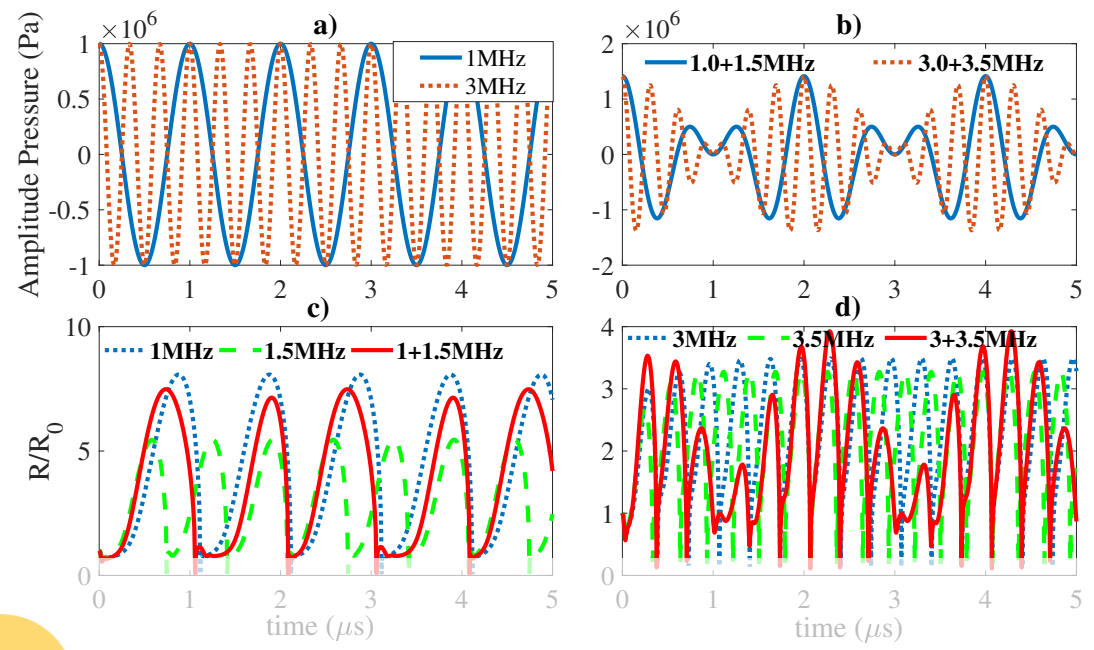

Figure 2: Behavior of acoustic signal for single- (a) and dual-frequency mode (b). The bubble dynamics in presence of single- and dual-frequency acoustic signal for low (c) and high frequencies (d)

bubble radius expansion is more clear (Figure 2d). Thus, for high frequencies, the application of the dual-frequency mode can lead to a lower value of the inertial cavitation threshold.

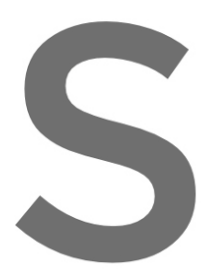

3.3 Inertial cavitation threshold
As was shown in the previous subsection, with the
can become larger compared to a single-frequency

in previous works $[9,11$, the use a dual-frequen
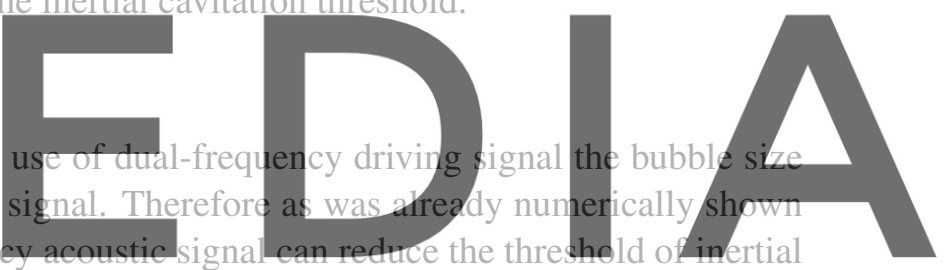

cavitation. To investigate the effect of dual-frequency excitation on the bubble dynamics two different

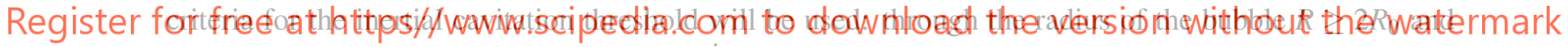
through the velocity of the bubble collapse $\dot{R} \leq-c$.

To determine the threshold value, the pressure value is increased using $1 \mathrm{kPa}$ increment, starting with 1 $\mathrm{kPa}$. We repeat this process for different initial values of the bubble radius (in the range from 0.1 to 10 $\mu \mathrm{m}$ with step size equals $0.01 \mu \mathrm{m}$ ) and for different combinations of frequencies.

Figure 3a shows the estimated threshold of inertial cavitation found using the radius criterion, whereas Figure $3 \mathrm{~b}$ shows the threshold determined using the velocity criterion of the bubble wall. As was mentioned before, in the dual-frequency mode, the threshold is lower than in the single-frequency mode, which is clearly seen for high frequencies. With an increase of initial values of the bubble, the threshold also starts to grow. Moreover, for low frequencies, this growth is not so sharp as for high frequencies. Different criteria of the threshold give different results.

Using the radius as the criterion gives a smaller prediction of the threshold than when using the velocity of the bubble collapse. Only values of the threshold for large bubbles in $10 \mu \mathrm{m}$ are approximately the same for both criteria. Thus, criterion with radius gives the lower limit of the threshold, and collapse velocity criterion gives the upper limit of the threshold value [26]. 

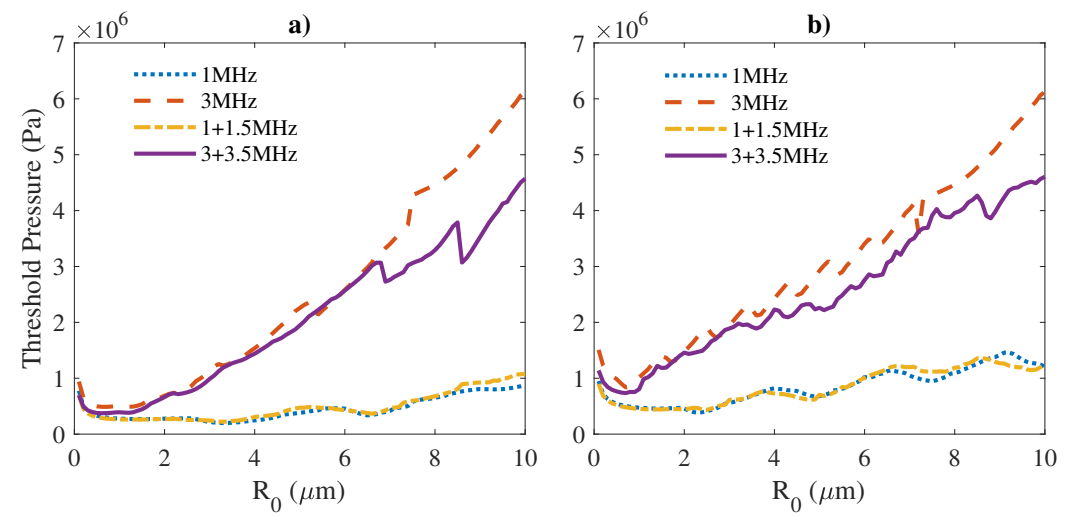

Figure 3: Predicted inertial cavitation threshold for single- and dual-frequency acoustic signal with threshold criteria $R \geq 2 R_{0}$ (a) and $\dot{R} \leq-c$ (b)

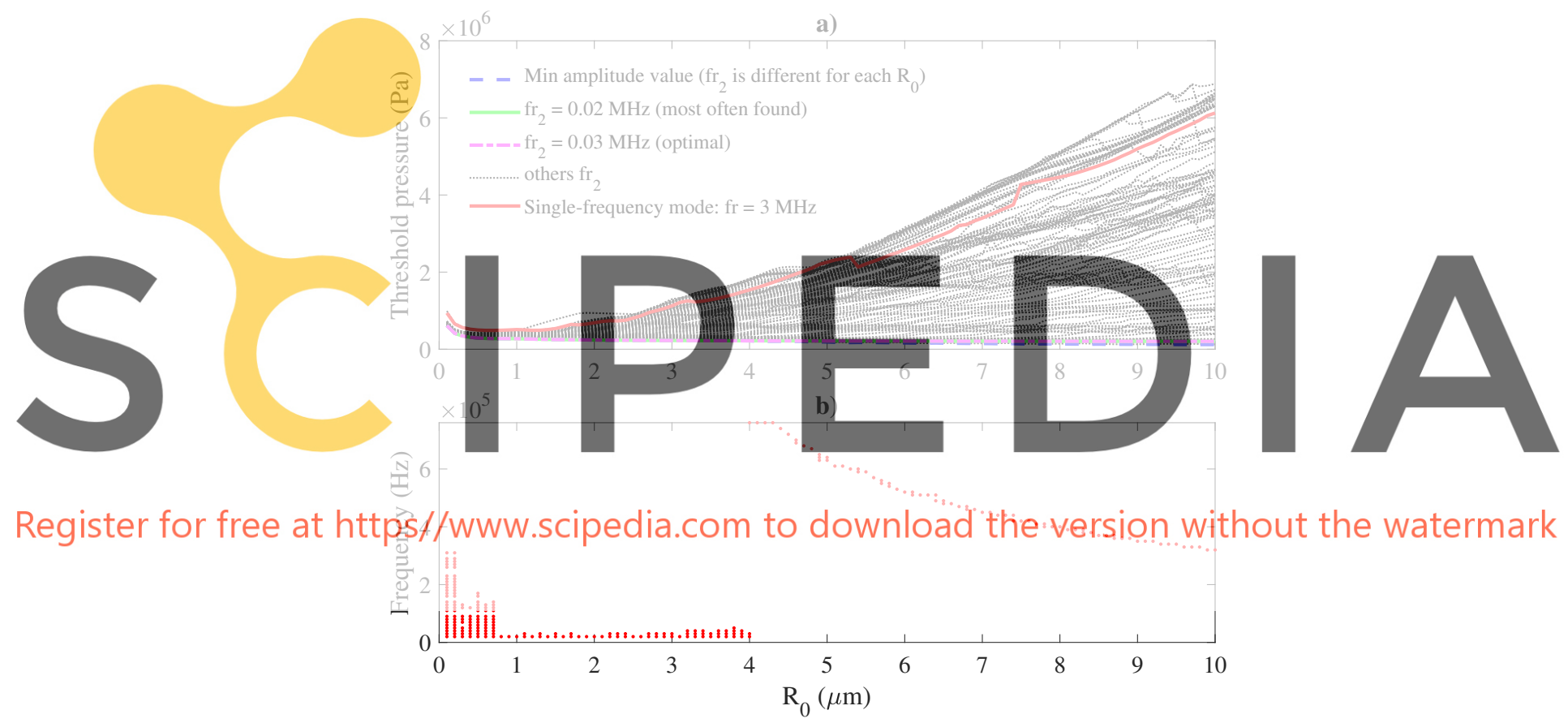

Figure 4: Predicted inertial cavitation threshold (a) and all optimal frequencies which together with fixed frequency at $3 \mathrm{MHz}$ lead to lower threshold pressure (b)

\subsection{Optimal frequency combinations}

Since different combinations of frequencies have different effects on the behavior of the bubble, and, on the threshold of inertial cavitation, in this paper one frequency has been fixed at $3 \mathrm{MHz}$. The value of another frequency has been varied in the range between $10 \mathrm{kHz}$ and $5 \mathrm{MHz}$ with step size equals $10 \mathrm{kHz}$. The results of all these combinations are analyzed and the frequency values that give the optimal (lowest) threshold of inertial cavitation for all considered initial values of the bubble radius are found (Figure 4a). 
Blue dashed line represents the lowest threshold pressure for each initial radius. It means that for different initial radius we have different optimal frequency. Thus, blue line is difficult to achieve since multiple bubbles with different radius can appear in tissue during HIFU therapy. However, this blue line can be used as a reference, in order to find the optimal second frequency for all bubbles sizes. This optimal frequency is equal $0.03 \mathrm{MHz}$, and the threshold is represented by magenta line. Red line shows threshold pressure when only one frequency is used ( $3 \mathrm{MHz}$ ).

Our results demonstrate that the big difference between two frequencies in a dual-frequency mode can help to sufficiently decrease the inertial cavitation threshold pressure.

Figure $4 \mathrm{~b}$ presents all frequency values for a given radius that help to achieve the minimum threshold of inertial cavitation. One can notice that the frequency, which the most often gives the minimum threshold pressure independent on the bubble size, is $0.02 \mathrm{MHz}$. These threshold results are represented by green line.

\section{CONCLUSIONS}

Gilmore-Akulichev-Zener model is a more general model for the investigation of bubble dynamics and inertial cavitation threshold in soft tissue since it is applicable for high Mach numbers $(M \leq$ 2.2).

- Inertial threshold criterion using radius $R \geq 2 R_{0}$ gives the lower threshold values in comparison with the criterion using bubble collapse velocity $\dot{R} \leq-c$.

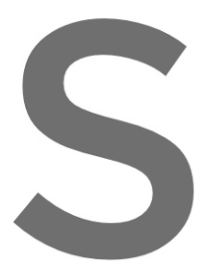

Dual-frequency signal mode can significantly reduce inertial cavitation threshold in comparison with single-frequency mode especially for high values of frequency ( $3 \mathrm{Mrz}$ and greater).

Threshold pressure pressure to start similar threshold p is chosen. The choice of laro value (for example, $3 \mathrm{MHz}$ and $0.03 \mathrm{MHz}$ ).

Register for free at https//www.scipedia.com to download the version without the watermark REFERENCES

[1] M. Solovchuk, T.W.H. Sheu, M. Thiniet, W.L. Lin. On a computational study for investigating acoustic streaming and heating during focused ultrasound ablation of liver tumor. Appl. Therm. Eng. (2013) 56:62-76.

[2] M. Solovchuk, M. Thiriet, T.W.H. Sheu. Computational study of acoustic streaming and heating during acoustic hemostasis. Appl. Therm. Eng. (2017) 124:1112-1122.

[3] K. Yasui. Acoustic Cavitation and Bubble Dynamics. Ultrasound and Sonochemistry. Springer (2018).

[4] R.G. Holt and R.A. Roy. Bubble dynamics in therapeutic ultrasound. In A.A. Doinikov (Ed.), Bubble and Particle Dynamics in Acoustic Fields: Modern Trends and Applications. Research Signpost, Kerala, India (2005).

[5] C.C. Coussios and R.A. Roy. Applications of acoustics and cavitation to noninvasive therapy and drug delivery. Annu. Rev. Fluid Mech. (2008) 40:395-420. 
[6] J.L. Laborde, C. Bouyer, J.P. Caltagirone, A. Gérard. Acoustic bubble cavitation at low frequencies. Ultrasonics (1998) 36:589-594.

[7] M. Ashokkumar, J. Lee, Y. Iida, K. Yasui, T. Kozuka, T. Tuziutib, A. Towata. The detection and control of stable and transient acoustic cavitation bubbles. Phys. Chem. Chem. Phys. (2019) 11:1011810121.

[8] X. Yang and C.C. Church. A model for the dynamics of gas bubbles in soft tissue. J. Acoust. Soc. Am. (2005) 118:3595-3606.

[9] M. Wang and Y. Zhou. Numerical investigation of the inertial cavitation threshold by dualfrequency excitation in the fluid and tissue. Ultrason. Sonochem. (2018) 42:327-338.

[10] F. Hegedüs, K. Klapcsik, W. Lauterborn, U. Parlitz, R. Mettin. GPU accelerated study of a dualfrequency driven single bubble in a 6-dimensional parameter space: The active cavitation threshold. Ultrason. Sonochem. (2020) 67:105067.

[11] D. Suo, B. Govind, S. Zhang, Y. Jing. Numerical investigation of the inertial cavitation threshold under multi-frequency ultrasound. Ultrason. Sonochem. (2018) 41:419-426.

[12] E. Zilonova, M. Solovchuk, T.W.H. Sheu. Bubble dynamics in viscoelastic soft tissue in highintensity focal ultrasound thermal therapy. Ultrason. Sonochem. (2018) 40:900-911.

[13] E. Zilonova, M. Solovchuk, T.W.H. Sheu. Simulation of cavitation enhanced temperature elevation in a soft tissue during high-intensity focused ultrasound thermal therapy. Ultrason. Sonochem. (2019) 53:11-24.

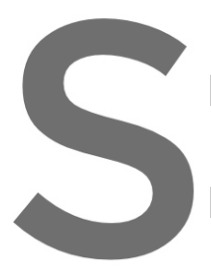

[14] C. Hua and E. Johnser linear viscoelastic

15] J. B. Keller and 68:628-633.
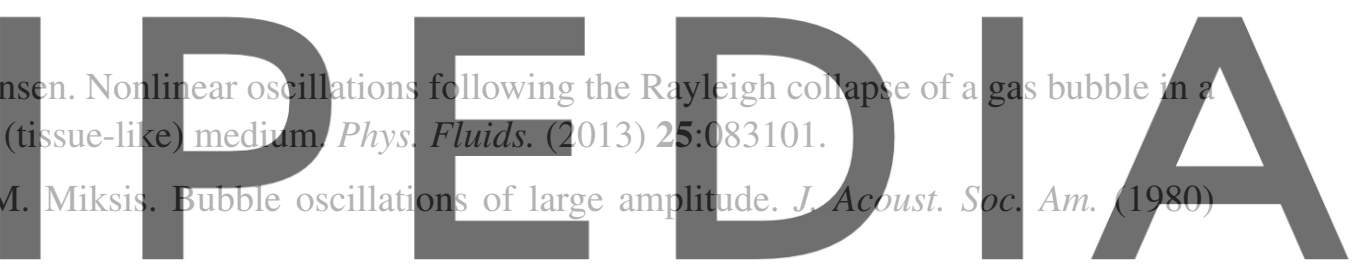

[16] A. Prosperetti and A. Lezzi. Bubble dynamics in a compressible liquid. Part 1. First-order theory.

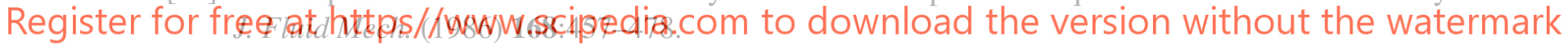

[17] A. Lezzi and A. Prosperetti. Bubble dynamics in a compressible liquid. Part 2. Second-order theory. J. Fluid Mech. (1987) 185:289-321.

[18] F.R. Gilmore. The growth or collapse of a spherical bubble in a viscous compressible liquid. Cal. Inst. Techn. Report No. 26-4 (1952).

[19] V.A. Akulichev. Pulsations of cavitation voids. In L.D. Rozenberg (Ed.), High-intensity ultrasonic fields (pp. 201-259). Ultrasonic Technology (A Series of Monographs). Springer, Boston, MA (1971).

[20] C.C. Church. A theoretical study of cavitation generated by an extracorporeal shock wave lithotripter. J. Acoust. Soc. Am. (1989) 86:215-227.

[21] K. Vokurka. Comparison of Rayleigh's, Herring's, and Gilmore's models of gas bubbles. Acta Acust. United Acust. (1986) 59:214-219.

[22] M. Solovchuk, T.W.H. Sheu, M. Thiriet. Simulation of nonlinear Westervelt equation for the investigation of acoustic streaming and nonlinear propagation effects. J. Acoust. Soc. Am. (2013) 
134:3931-3942.

[23] M. Solovchuk, T.W.H. Sheu, M. Thiriet. Multiphysics modeling of liver tumor ablation by high intensity focused ultrasound. Commun. Comput. Phys. (2015) 18:1050-1071.

[24] C.C. Church. Frequency, pulse length, and the mechanical index. Acoust. Res. Lett. Online (2005) 6:162-168.

[25] C.C. Church, C. Labuda, K. Nightingale. A theoretical study of inertial cavitation from acoustic radiation force impulse imaging and implications for the mechanical index. Ultrasound Med. Biol. (2015) 41:472-485.

[26] I.R. Webb, S.J. Payne, C.-C. Coussios. The effect of temperature and viscoelasticity on cavitation dynamics during ultrasonic ablation. J. Acoust. Soc. Am. (2011) 130:3458-3466.

[27] H.G. Flynn. Cavitation dynamics. II. Free pulsations and models for cavitation bubbles. J. Acoust. Soc. Am. (1975) 58:1160-1170.

[28] J.R. Dormand, P.J. Prince. A family of embedded Runge-Kutta formulae. J. Comput. Appl. Math. (1980) 6:19-26.
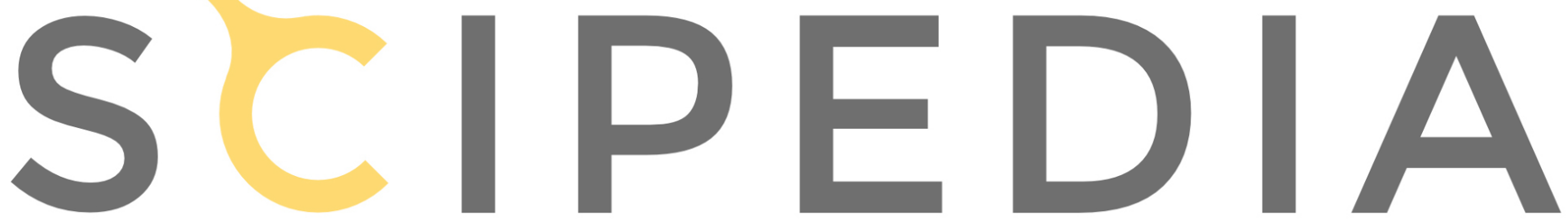